\title{
Compliance with bladder management in spinal cord injury patients
}

\author{
G Yavuzer*,1, H Gök ${ }^{1}$, S Tuncer ${ }^{1}$, T Soygür ${ }^{2}$, N Arikan ${ }^{2}$ and T Arasil ${ }^{1}$ \\ ${ }^{1}$ Department of Physical Medicine and Rehabilitation, Ankara University Medical School, Ankara, Turkey; \\ ${ }^{2}$ Department of Urology, Ankara University Medical School, Ankara, Turkey
}

Study design: Retrospective analysis of medical records on spinal cord injury (SCI) patients with neuropathic bladder.

Objective: To determine SCI patients' compliance with the method of bladder management they used on discharge from inpatient rehabilitation.

Setting: Ankara University Medical School, Department of Physical Medicine and Rehabilitation, Spinal Cord Injury Unit, which treats patients referred from throughout Turkey.

Methods: The bladder management method of 50 new SCI patients was noted at admission, discharge and follow-up. Reasons for changing the initial method were documented. For analysis, patients were grouped by gender, level and completeness of injury. Compliance with bladder management method was compared between these groups by chi-square test.

Results: The method of bladder management at admission was indwelling catheter (IC) for $86 \%$ of the patients. Most were switched to clean intermittent catheterisation (CIC) by rehabilitation discharge. Of 38 patients $(76 \%)$ on CIC at discharge, $20(52 \%)$ discontinued this method and reverted to IC during follow-up. Compliance with CIC was lower for women, for tetraplegics, and for those with complete injury. Dependence on care givers, severe spasticity interfering with catheterisation, incontinence despite anticholinergic agents, and lack of availability of external collective devices for female patients were the main reasons for low compliance with CIC.

Conclusion: The bladder management method of SCI patients should be selected so as to be suitable to the patients' life style. Besides reducing morbidity, it also has to enhance the quality of life.

Spinal Cord (2000) 38, $762-765$

Keywords: spinal cord injury; neuropathic bladder; bladder management; tetraplegia; rehabilitation

\section{Introduction}

The management of neuropathic bladder dysfunction is a crucial component of a spinal cord injury (SCI) rehabilitation program. The loss of normal bladder function in patients with SCI is disabling and results in increased risk of urinary tract deterioration, giving rise to significant morbidity and occasionally to mortality. ${ }^{1}$ With a proper bladder management method that optimizes both renal function and social functioning, the person with a spinal cord injury can enjoy a much healthier life.

Our standard neuropathic bladder protocol has been designed to accomplish that aim. Patients admitted to the rehabilitation unit on indwelling catheter (IC) were switched during their stay, if possible, to sterile intermittent catheterisation (CIC). Bladder emptying was managed by sterile intermittent (self) catheterisation using a sterile No. 14 Nelaton catheter 4-6 times a day. During the inpatient period,

*Correspondence: G Yavuzer, Mustafa Kemal Mahallesi, Bariş Sitesi 87. sokak No 24, 06530 Ankara, Turkey patients were taught clean CIC, and they switched upon discharge. They were instructed to reuse, after discharge, catheters which are soaked in dilute aqueous Hibitane. Patients with a reflex bladder and sustained high intravesical pressures were placed on oral anticholinergic medication. Urine analysis, creatinine clearance, ultrasonic evaluations of the urinary tract, intravenous pyelography, urodynamic evaluations (including water cystometry) and sphincter EMG studies were performed while the patient was on the ward, and regularly at follow-up.

However, our informal observations suggested that during follow-up quite a few of the patients who had been discharged on CIC changed their bladder management method on their own, back to IC. This suggested that our protocol resulted in management methods that were not optimal from the patients' point of view. We decided to investigate this issue using review of patients' charts.

The aim of the present study is to determine SCI patients' compliance with the method of bladder 
management they used on inpatient rehabilitation discharge. The reasons for lack of compliance with CIC were also documented.

\section{Patients and methods}

The medical records of 50 new SCI patients admitted to the Ankara University Medical School, Department of Physical Medicine and Rehabilitation, Spinal Cord Injury Unit from 1994 to 1997 were reviewed. The following was abstracted: gender and age of the patient, level and completeness of injury, time since injury upon rehabilitation admission; length of stay in the rehabilitation unit; results of complete physical, neurological and urological examinations, American Spinal Injury Association (ASIA) Impairment Scale class at admission, discharge and follow up.

The method of bladder management the patient used was also noted, at admission, discharge and at every follow-up. Reasons for changing the initial method were documented.

These data were entered into a computer database and analyzed using the Statistical Package for the Social Sciences (SPSS) ${ }^{2}$ software program. Descriptive statistics of the patients' demographic and clinical features were prepared. For analysis, patients were grouped by gender, injury level (tetraplegia vs paraplegia) and completeness of injury (ASIA Impairment Scale Class A vs B-D). Rates of follow-up compliance with the bladder management method the patient was utilizing at discharge were compared between these groups using the chi-square test.

\section{Results}

Demographic and clinical characteristics of the 50 new SCI patients who were referred for inpatient rehabilitation are presented in Table 1. The mean age was 38 years (standard deviation (SD): 15 years). Twenty-eight per cent of the group were women. The average time since injury was 124 (SD 80 days), rehabilitation length of stay (LOS) was 130 (SD 50) days, and the average length of the follow-up period was 24 (SD8) months. Seven patients $(14 \%)$ had tetraplegia and $43(86 \%)$ paraplegia. Twenty-six patients had complete injuries $(52 \%)$ and 24 patients incomplete lesions (ASIA Classes B-D).

Urodynamic evaluations revealed that most of the patients $(82 \%)$ had an areflexic bladder at admission, whereas hyperreflexia was the prevalent finding $(64 \%)$ at discharge. At discharge, detrusor sphincter dyssynergia (DSD) was observed in only five patients $(10 \%)$.

The initial (admission) method of bladder management was indwelling catheter (IC) for $86 \%$ of the patient group. At discharge, CIC was the most common method $(74 \%)$; the majority of those patients on CIC were partially independent in self care, usually requiring some assistance for dressing and/or set up of the equipment for self-catheterising.
Follow-up data revealed a significant rate of change in the method of bladder drainage. Of the 38 patients on CIC at discharge, $20(52 \%)$ had discontinued this method and reverted to IC by an average of 24 months post discharge. Sixteen $(42 \%)$ continued on CIC and two patients $(6 \%)$ regained spontaneous micturation.

The percentage of patients in each group who reverted from CIC to IC after discharge is shown in Table 2. We observed that compliance with CIC was lower for tetraplegic patients (chi-square $=23.0$, $P<0.05)$. The rate of reverting to IC was $80 \%$ for tetraplegics and $49 \%$ for paraplegics.

Ten of the 14 female patients were discharged on CIC, and six $(60 \%)$ of them reverted to IC during follow-up. Twenty-eight of the 36 male patients were discharged on CIC. Their rate of reverting to IC was $50 \%$. Although the difference was not statistically significant $\quad($ chi-square $=11.4, \quad P>0.05), \quad$ compliance with the CIC program was lower for female patients.

Twenty-two of 26 patients with an ASIA Impairment Scale class A were discharged on CIC. Fifteen of

Table 1 Demographic and clinical characteristics of the patients

\begin{tabular}{|c|c|c|c|}
\hline & $\mathrm{n}$ & $\begin{array}{l}\text { Mean } \\
(S D)\end{array}$ & Range \\
\hline Age (years) & & $38(15)$ & $18-58$ \\
\hline $\begin{array}{l}\text { Time since injury } \\
\text { (days) }\end{array}$ & & $124(80)$ & $10-210$ \\
\hline $\begin{array}{l}\text { Rehabilitation length of } \\
\text { stay (days) }\end{array}$ & & $130(50)$ & $40-190$ \\
\hline $\begin{array}{l}\text { Duration of follow-up } \\
\text { (months) }\end{array}$ & & $24(8)$ & $5-40$ \\
\hline \multicolumn{4}{|l|}{ Gender } \\
\hline Female & 14 & & \\
\hline Male & 36 & & \\
\hline \multicolumn{4}{|l|}{ Level of injury } \\
\hline Tetraplegia & 7 & & \\
\hline Paraplegia & 43 & & \\
\hline \multicolumn{4}{|c|}{ ASIA impairment scale class } \\
\hline Class A & 26 & & \\
\hline Class $\mathrm{B}-\mathrm{D}$ & 24 & & \\
\hline
\end{tabular}

Table 2 Per cent of patients who at last follow-up had reverted from clean intermittent catheterisation to indwelling catheter, by gender, level and completeness of injury

\begin{tabular}{lll}
\hline Gender & Female & $60 \%$ \\
& Male & $50 \%$ \\
Level of injury & Tetraplegia & $80 \%$ \\
& Paraplegia & $49 \%$ \\
Asia impairment scale class & Class A & $68 \%$ \\
& Class B-D & $31 \%$ \\
Total reverted to indwelling & & $52 \%$ \\
catheter & &
\end{tabular}


them reverted to IC (rate of $68 \%$ ). Only five of the 16 patients with incomplete injuries changed their method from CIC to IC. The rate was $31 \%$ and the difference was statistically significant (chi-square $=34.3$, $P<0.01)$.

The major reasons for changing the method were reported as: dependence on care givers, severe spasticity interfering with catheterisation, incontinence despite anticholinergic agents and, for female patients, inconvenience of external collective devices.

\section{Discussion}

In Turkey, the intervention for bladder dysfunction during the acute stage of SCI is usually the indwelling catheter or use of a cystocatheter. The choice of a chronic drainage method beyond the early stage depends on a variety of factors, including type of bladder dysfunction, patient preference and physician bias.

In the 1970s Lapides et al first promoted long-term $\mathrm{CIC}^{3}$ and since then CIC has gradually gained acceptance. It has become the standard procedure for managing the neuropathic bladder of SCI patients. ${ }^{4,5}$ Recently, Weld et al presented CIC as the safest and most appropriate method of bladder management for these patients. ${ }^{6,7}$ Prior to this study, our policy with respect to spinal cord injured patients who did not void spontaneously was to put them on long term CIC, enabling them to be continent and catheter free. The final decision was always made after discussing the pros and cons of the options of bladder management, with the patient, and his or her family caregivers.

In our study the prevalent bladder management method at admission to the rehabilitation unit was found to be IC $(86 \%)$, which commonly was replaced by CIC at discharge ( $74 \%$ of the 43 patients on CIC). At a mean follow-up duration of 24 months after discharge, more than half of the patients $(52 \%)$ who had been discharged on CIC had changed in favour of IC. Compliance with CIC was lower in our study than reported by some other authors. Gallien et $a l,{ }^{8}$ Maynard and Glass ${ }^{9}$ and Hill et $a l^{5}$ reported the rate of changing from CIC to IC as $6 \%, 6 \%$ and $15 \%$, respectively. Maynard and Diokno reported the compliance with CIC as $86 \% .^{10}$ On the other hand, Bakke et al observed aversion to CIC by one third of their patients. ${ }^{11}$ Timoney and Shaw noted that only $50 \%$ of their female patients could continue on their CIC program. ${ }^{12}$

Vaidyanathan et al described the perfect treatment of neuropathic dysfunction of the urinary bladder to be that one which helps the patient to simulate as closely as possible the life style of an able-bodied individual. ${ }^{13}$ Our data, based on periodic evaluations, allowed us to determine that the identity of the bladder management method which was chosen as best during discussions on the ward may change after discharge. The low compliance of our SCI patients with CIC raises the question whether the long-term options were inadequately managed by us, and whether there are some other parameters than renal function and urinary tract morbidity that have to be taken into consideration before deciding on a patient's bladder management program. The main reason for changing the method of bladder management could be the changing life style of the patients.

The main reasons for keeping a patient on IC during the stay on the rehabilitation unit (rather than switching her or him to CIC) were spasticity, the patients' psychological condition, low bladder capacity with high detrusor pressures, inadequate effects of anticholinergic drugs, pressure ulcers and prior complications like ureterovesical reflux or hydronephrosis. Dependence on caregivers and unacceptable incontinence were the principal reasons patients gave for changing their bladder management method from CIC to IC during follow-up.

Because they were more often dependent on selfcatheterisation and more often incontinent (having no chance of using external collectors), $60 \%$ of the female patients reverted from CIC to IC. The selection of the most appropriate method of bladder management for females with reflex bladders may need to rely more on psychosocial considerations than on concern about preservation of renal function. ${ }^{14}$ In the study by Bakke et $a l$, dissatisfaction with and aversive reactions to CIC were reported by more women than men. ${ }^{11}$ In our study, continued use rates of CIC in females with SCI are lower than in men. The difference in this series is due to persistence of incontinence refractory to anticholinergic suppression. ${ }^{12,15}$ During long-term follow-up, we observed that some of the female patients who needed to be catheterised by someone else started to perceive the procedure as a sexual assault. Usually this occurred after the first 2 years during which they thought that catheterisation would be temporary, and that they were again going to urinate by themselves one day. However, male patients indicated that they wanted to feel ready for sexual interaction with their partner whenever the correct time would come, without an indwelling catheter getting in the way. The same desire has been reported by men with SCI in England. ${ }^{13}$

Eight per cent of our tetraplegic patients preferred a long-term indwelling urethral catheter, as they found this method to be practical and socially preferred. Spasticity interfering with intermittent catheterisation, inconvenience, limitations of hand function, and high dependence on care givers were the main reasons for these patients. The incidence of urological complications in tetraplegic patients with and without indwelling catheter have been found to be similar. ${ }^{16}$ Our results indicate that for tetraplegic patients, there appear to be few alternatives to indwelling catheters.

In conclusion, intermittent catheterisation may be the preferred option for well-motivated and cooperative male paraplegic patients with good hand function. For female tetraplegic patients with poor hand 
function, spasticity or incontinence, IC recommends itself. Patients and family caregivers should play a role in the selection of a management method, and the final decision should depend on the patients' comfort, convenience and quality of life. For selected SCI patients who start community living using CIC, an indwelling catheter may be considered if the first choice fails.

\section{References}

1 Menon EB, Tan ES. Bladder training in patients with spinal cord injury. Urology 1992; 40: 425-429.

2 Statistical Package for the Social Sciences (SPSS) for Windows, Version 7.0; SPSS Inc., 444 N. Michigan Avenue, Chicago, IL.

3 Lapides $\mathbf{J}$ et al. Clean intermittent self-catheterization in the treatment of urinary tract disease. J Urol 1972; 107: 458-465.

4 Kuhn W, Rist M, Zaech GA. Intermittent urethral self catheterisation: Long term results (Bacteriological evolution, continence, acceptance, complications). Paraplegia 1991; 29: $222-232$.

5 Hill VB, Davies WE. A swing to intermittent clean self catheterisation as a preferred mode of management of the neuropathic bladder for the dextrous spinal cord patient. Paraplegia 1988; 26: 405 - 412.

6 Weld KJ, Dmochowski RR. Effect of bladder management on urological complications in spinal cord injured patients. $J$ Urol 2000; 163: $768-772$

7 Weld KJ, Graney MJ, Dmochowski RR. Differences in bladder compliance with time and associations of bladder management with compliance in spinal cord injured patients. J Urol 2000; 163: $1228-1233$.
8 Gallien $\mathrm{P}$ et al. Influence of urinary management on urologic complications in a cohort of spinal cord injury patients. Arch Phys Med Rehabil 1998; 79: 1206-1209.

9 Maynard FM, Glass J. Management of the neuropathic bladder by clean intermittent catheterisation: 5 year outcomes. Paraplegia 1987; 25: $106-110$.

10 Maynard FM, Diokno AC. Clean intermittent catheterisation for spinal cord injured patients. J Urol 1982; 128: 477-480.

11 Bakke A, Irgens LM, Malt UF, Hoisaeter PA. Clean intermittent catheterisation - performing abilities, aversive experiences and distress. Paraplegia 1993; 31: 288-297.

12 Timoney AG, Shaw PJR. Urological outcome in female patients with spinal cord injury: The effectiveness of intermittent catheterisation. Paraplegia 1990; 9: 556- 563.

13 Vaidyanathan $\mathrm{S}$ et al. Effect of intermittent urethral catheterisation and oxybutynin bladder instillation on urinary continence status and quality of life in a selected group of spinal cord injury patients with neuropathic bladder dysfunction. Spinal Cord 1998; 36: $409-414$.

14 Stover SL et al. Urological management and follow-up of spinal cord injured females. Paraplegia 1983; 21: 184 (Abstract).

15 Barnes DG, Shaw PJR, Timoney AG, Tsokos N. Management of the neuropathic bladder by suprapubic catheterization. Br J Urol 1993; 72: $169-172$.

16 Dewire DM et al. A comparison of the urological complications associated with long-term management of quadriplegics with and without chronic indwelling urinary catheters. J Urol 1992; 147: $1069-1072$. 